\title{
Trained and Untrained Female Singers Using Electroglottography Parameters: A Comparative Study
}

\author{
Chandan Biswas ${ }^{1}$, Indranil Chatterjee ${ }^{2}$, Joyanta C Mandal ${ }^{3} \odot$
}

\begin{abstract}
Aim and objective: This study is aimed to evaluate and compare the effect of training on female trained (Hindustani classical) singers and untrained singers using electroglottography (EGG) parameters.

Materials and methods: A total of 60 female subjects aged between 18 and 30 years were categorized into two groups: 30 female Hindustani classical singers and 30 untrained female singers. EG-PC3 EGG system of Dr. Speech software, TigerDRS Inc was used for this study. The following five parameters were taken into consideration: contact quotient $(\mathrm{CQ})$, contact index $(\mathrm{Cl})$, contact quotient perturbation (CQP), contact index perturbation (CIP), and contact ratio (CR). The recording procedure involved two tasks: singing and speech.

Results: By comparing these two groups with t-test, it has been found that $p$ value is less than 0.05 (here $a=0.05$ level of significance, $95 \%$ confidence level) for all the parameters which revealed significant difference of EGG parameters in trained and untrained singers for singing task as well as for speech task.

Conclusion: All the parameters are proved to be useful to show differences. The quantitative parameters with higher significance to differentiate were $\mathrm{CQ}, \mathrm{Cl}, \mathrm{CQP}, \mathrm{CIP}$, and $\mathrm{CR}$, which provide information with respect to the glottis contact, the vibratory regularity, the vibration symmetry, and the speed of closing and opening of the vocal folds.

Clinical significance: The study would help professional voice users to understand the impact of training on their phonatory capability and on their everyday speech pattern, thereby providing vocal education to the singers to prevent vocal abuse especially in case of untrained singers. Keywords: Electroglottography, Trained singer, Untrained singer, Vocal folds, Voice.

International Journal of Phonosurgery \& Laryngology (2021): 10.5005/jp-journals-10023-1221
\end{abstract}

\section{INTRODUCTION}

The physiology of vocal music is very complex. Vocal performers train and practice to produce aesthetically pleasing sound. In its physical aspect, singing has a well-defined technique that depends on the use of the respiratory system, phonatory system, resonatory system, and articulatory system that impose consonants and vowels on the amplified sound. Though these four mechanisms function independently, they are nevertheless coordinated in the establishment of a vocal technique and are made to interact upon one another. Sound also resonates within different parts of the body, and an individual's size and bone structure can affect somewhat the sound produced by an individual. Singers can also learn to project sound in certain ways so that it resonates better within their vocal tract. This is known as vocal resonation.

Singers amongst professional voice users have the greatest vocal demands and require the most sophisticated analysis. However, the term trained singer, untrained singer, and nonsinger are not bound by a set of precisely well-defined criteria. Akerlund and Gramming are considered trained singers, who had 4 years of training and 10 years of experience of stage and concert and nonsingers as those individuals who had no exposure to formal training in speech or music. ${ }^{1}$ Awan considered trained singers as individuals who have been in formal voice training program from a period of at least 2 years and considered untrained singers as individuals with casual singing experience. ${ }^{2}$ According to Ghosh, trained singers have an exposure to formal training for a period of 10 years, whereas untrained singers practiced folklore/film songs on a regular basis without any exposure to formal voice training. ${ }^{3}$
${ }^{1}$ Rise Speech and Hearing rehabilitation Centre, Kolkata, West Bengal, India

${ }^{2}$ Department of Speech Language Pathology, Ali Yavar Jung National Institute for the Hearing Handicapped, ERC, Kolkata, West Bengal, India ${ }^{3}$ Speech and Language Diagnostic Department, Ali Yavar Jung National Institute of Speech and Hearing Disabilities ERC, Kolkata, West Bengal, India

Corresponding Author: Joyanta C Mandal, Speech and Language Diagnostic Department, Ali Yavar Jung National Institute of Speech and Hearing Disabilities ERC, Kolkata, West Bengal, India, Phone: +91 8240117189, e-mail: joyantachmandal@gmail.com

How to cite this article: Biswas C, Chatterjee I, Mandal JC. Trained and Untrained Female Singers Using Electroglottography Parameters: A Comparative Study. Int J Phonosurg Laryngol 2021;11(2):33-38.

Source of support: Nil

Conflict of interest: None

Electroglottography (EGG) uses the movement induced by variations in the electric impedance and supplies information about the vibratory patterns of the vocal folds in a noninvasive way, what is highly useful since it permits the evaluation of the patient in phonation physiological conditions. The usefulness of this technique is worldwide acknowledged both in vocal clinic and in research. It is being recently applied in education, especially in the sung voice. ${ }^{4}$ The EGG measures the variation in impedance to a very small electrical current between the electrodes pair placed across the neck as the area of vocal fold contact changes during voicing.

\footnotetext{
(c) The Author(s). 2021 Open Access This article is distributed under the terms of the Creative Commons Attribution 4.0 International License (https://creativecommons. org/licenses/by-nc/4.0/), which permits unrestricted use, distribution, and non-commercial reproduction in any medium, provided you give appropriate credit to the original author(s) and the source, provide a link to the Creative Commons license, and indicate if changes were made. The Creative Commons Public Domain Dedication waiver (http://creativecommons.org/publicdomain/zero/1.0/) applies to the data made available in this article, unless otherwise stated.
} 


\section{Need of the Study}

At present, very limited study review focusing solely on the impact of voice training in professional voice users on EEG parameters in Indian scenario. So it is very much essential to investigate the impact of voice training among professional voice users. In the aspect of speech science, EGG parameterization for classical singers should be taken up to establish a reference in terms of normative for the amount of changes possible to make the vocal pathogenesis of two groups of singers, mainly trained and untrained singers. Current studies are predominantly based on the Carnatic/Karnatic style of classical singing, so need to focus on Hindustani classical singing also for new dimension.

\section{Aim and Objective}

The present study is aimed to evaluate the effect of training on EGG parameters and to compare EGG parameterization between female trained (Hindustani classical) singers and untrained singers and to validate whether EGG is a unique tool to differentiate vocal fold vibratory characteristics between trained and untrained singers.

\section{Materials and Methods}

A total of 60 female subjects aged between 18 and 30 years were taken for the study and categorized into two groups. The categories are trained singers which consisted of 30 [mean age $=22.74$ years, standard deviation $(S D)=6.9$ ] female Hindustani classical singers and untrained singers consisted of 30 (mean age $=22.16$ years, $S D=5.3$ ) female singers. The participants who had no chronic medical problems or any history of voice problems for the last 3 months, no laryngeal structural problem, no respiratory tract infection during assessment, no history of smoking, and had normal hearing and speech language abilities were selected for this study.

EG-PC3 EGG system of Dr. Speech software (Version-4), Tiger DRS Inc was used for this study. Sampling rate was $44,100 \mathrm{~Hz}$ and the recording time was selected for 3 to 5 seconds. Parameters of EGG will set in the Dr. Speech program. EGG calibration was done according to the specification of Tiger Electroglottograph: EG-PC3. The skin of the neck was prepared by rubbing with skin prep gel. The electrodes, mounted on a Velcro strip, were smeared with an electrode conductance gel and then put on the neck tightly, so that the two electrodes lie overhanging the thyroid lamina. Recording time period was set for 3 seconds in speech and 5 seconds for singing task in time window. Five parameters were taken into consideration: contact quotient $(\mathrm{CQ})$, contact index $(\mathrm{CI})$, contact quotient perturbation (CQP), contact index perturbation $(\mathrm{CIP})$, and contact ratio (CR).

The recording procedure involved two tasks: singing and speech. In the singing task, the subjects were asked to produce the vowel/æ/ at their habitual frequency and at comfortable loudness level. The corresponding musical scale /do-re-me/ to their habitual frequency was noted as the reference tone which was used further in the procedure. The reference tone was again played out to the subject. The subject matched the scale of the reference tone at her comfortable loudness level. For maintaining the lowest level of loudness, the subject increased the pitch through the musical scales /do -re- me/ till the highest pitch level was reached. And in speech task is determined by asking the subject to phonate /a/ vowel at comfortable loudness level.

\section{Results}

Descriptive statistics of EGG parameters for both the groups in two different tasks was chosen for the present study (mean, standard deviation, minimum value, and maximum value). Mean and SD of each parameters for untrained singers are given in Table 1 and for trained singers in Table 2.

Two-sample paired t-test was carried out to check whether any significant difference exists between the untrained and trained singers in both singing and speech task using EEG parameters. The computed results are presented in Table 3 for singing task and in Table 4 for speech task.

By comparing these two groups with t-test, it has been found that $p$ value is less than 0.05 (here at $a=0.05$ level of significance, 95\% confidence level) for all the parameters (CQ, Cl, CQP, CIP, and CR) which revealed significant difference of EGG parameters in trained and untrained singers for singing task as well as speech task.

\section{Discussion}

Contact Quotient (Singing task): The EGG CQ is sometimes also referred to as "Closed Quotient" or "EGG Closed Quotient". The CQ is a ratio of the contact period to the total duration of one cycle of adduction and abduction. ${ }^{5}$ The CQ is used to compare the duration of the contact phase to the period of the vibratory cycle. ${ }^{6}$ In this study, mean CQ was 78.44 for a trained female singer which was higher than untrained female singers (73.44) in singing task.

Contact quotient depends on the rate of adduction and abduction of the vocal folds. It is assumed that higher values of $\mathrm{CQ}$ in trained singers have higher rate of glottis abduction and adduction. In singing task, the rate is modulated by higher mean values of $C Q$ observed in singing task for both trained and untrained

Table 1: Mean and SD of each EGG parameters for untrained singers

\begin{tabular}{|c|c|c|c|c|c|}
\hline Parameters & Tasks & Mean & Standard deviation & Min & $\operatorname{Max}$ \\
\hline \multirow[t]{2}{*}{ Contact quotient } & Singing task & 73.44 & 0.84 & 52.32 & 80.24 \\
\hline & Speech task & 67.91 & 1.64 & 48.61 & 81.30 \\
\hline \multirow[t]{2}{*}{ Contact index } & Singing task & -0.25 & 0.03 & -0.11 & -0.47 \\
\hline & Speech task & -0.14 & 0.01 & -0.08 & -0.29 \\
\hline \multirow[t]{2}{*}{ Contact quotient perturbation } & Singing task & 3.92 & 0.55 & 0.90 & 5.71 \\
\hline & Speech task & 2.95 & 0.48 & 0.71 & 4.92 \\
\hline \multirow[t]{2}{*}{ Contact index perturbation } & Singing task & 10.30 & 3.63 & 7.85 & 15.13 \\
\hline & Speech task & 9.37 & 1.11 & 6.03 & 15.44 \\
\hline \multirow[t]{2}{*}{ Contact ratio } & Singing task & 59.99 & 0.89 & 45.67 & 66.27 \\
\hline & Speech task & 46.46 & 2.52 & 39.06 & 61.34 \\
\hline
\end{tabular}


Table 2: Mean and SD of each EGG parameters for trained singers

\begin{tabular}{|c|c|c|c|c|c|}
\hline Parameters & Subjects & Mean & Standard deviation & Min & Max \\
\hline \multirow[t]{2}{*}{ Contact quotient } & Singing task & 78.44 & 1.59 & 50.03 & 88.13 \\
\hline & Speech task & 76.29 & 1.06 & 51.14 & 84.77 \\
\hline \multirow[t]{2}{*}{ Contact index } & Singing task & -0.36 & 0.03 & -0.13 & -0.68 \\
\hline & Speech task & -0.27 & 0.04 & -0.10 & -0.55 \\
\hline \multirow[t]{2}{*}{ Contact quotient perturbation } & Singing task & 3.03 & 0.28 & 1.01 & 5.39 \\
\hline & Speech task & 1.73 & 0.24 & 0.82 & 3.34 \\
\hline \multirow[t]{2}{*}{ Contact index perturbation } & Singing task & 9.39 & 1.50 & 5.44 & 13.21 \\
\hline & Speech task & 7.98 & 2.96 & 4.92 & 11.65 \\
\hline \multirow[t]{2}{*}{ Contact ratio } & Singing task & 63.82 & 0.71 & 42.80 & 77.14 \\
\hline & Speech task & 55.29 & 1.52 & 43.71 & 67.29 \\
\hline
\end{tabular}

Table 3: Comparison of EGG parameters between untrained and trained singers in singing task

\begin{tabular}{lllrcc}
\hline Subjects & Parameters & $d f$ & $t$-value & $p$-value & Interpretation \\
\hline Untrained and trained singers & Contact quotient & 58 & -15.26 & 0.00 & Significant difference \\
Untrained and trained singers & Contact index & 58 & -15.13 & 0.00 & Significant difference \\
Untrained and trained singers & Contact quotient perturbation & 58 & -7.79 & 0.00 & Significant difference \\
Untrained and trained singers & Contact index perturbation & 58 & -12.77 & 0.00 & Significant difference \\
Untrained and trained singers & Contact ratio & 58 & -18.39 & 0.00 & Significant difference \\
\hline
\end{tabular}

Table 4: Comparison of EGG parameters between untrained and trained singers in speech task

\begin{tabular}{llllcc}
\hline Subjects & Parameters & $d f$ & $t$-value & $p$-value & Interpretation \\
\hline Untrained and trained singers & Contact quotient & 58 & -18.15 & 0.00 & Significant difference \\
Untrained and trained singers & Contact index & 58 & -23.54 & 0.00 & Significant difference \\
Untrained and trained singers & Contact quotient perturbation & 58 & -12.57 & 0.00 & Significant difference \\
Untrained and trained singers & Contact index perturbation & 58 & -37.89 & 0.00 & Significant difference \\
Untrained and trained singers & Contact ratio & 58 & -13.29 & 0.00 & Significant difference \\
\hline
\end{tabular}

singer's voice. This can be assumed that trained singer's voice used a technique which is known as "Vibrato." It is the oscillation and modulation of sustained tone. A healthy vibrato occurs at the rate of six to eight oscillations per second in singers, which is higher than the normal individuals. It is commonly used in all styles of music, and is great for placing an emphasis on significant words of a musical piece. ${ }^{7}$ Thus CQ values are assumed to be higher due to more oscillations of the vocal folds.

Howard studied on closed quotient through EGG measures to differentiate between trained and untrained female singers with the task vowel/a/sung by trained and untrained singers. Results showed CQ values occurred in the higher pitches above B4 in trained singers group. Therefore, it was suggested that $C Q$ values could be useful parameters to determine efficiency of singing training as it is related to the higher oscillation of vocal folds. Glottis abduction and adduction rates were varied with reference to different vowels' production. ${ }^{8}$

Wang and Zhang measured CQ in acoustic test of vowels like /e, /a/, /o/, which were sung by 10 opera singers aged between 20 and 64 years and measured through Dr. Speech version 4.0 software and result showed statistical significance of $C Q$ values in different vowels singing task. ${ }^{9}$

Contact Quotient (Speech task): Contact quotient mean (76.29) of trained female singers was also higher than CQ mean (67.91) of untrained female singers in speech task. The significant differences of $C Q$ in aspects of singer's voice can be explained in terms of laryngeal physiology. Contact quotient in singing training/experience could be interpreted in terms of a more physically efficient voice usage as follows: (a) the time for which an acoustic path to the lungs via an open glottis exists is reduced, resulting in a reduction in the total acoustic energy transmitted to the essentially anechoic environment of the lungs (known as subglottal damping) where it would be lost to the listener, (b) less stored lung air is vented in each cycle due to the decreased open phase, thereby improving the efficiency of power source usage and enabling notes to be held for a longer time, and (c) the perceived voice quality is less breathy. ${ }^{8}$

The increased CQ values in trained singers may be effects of training on singing and the experiences of vocal exercises. Cecconello, Salvador, and Córdoba analyzed the efficiency of qualitative and quantitative parameters of the EGG in among different singing style and experience. It was found higher values of CQ in the group of higher singing exposure. ${ }^{10}$

Contact quotient values must be decreased according to aging due to physiological changes of vocal fold apertures. Watson and Bier studied the significance of $C Q$ values in different vowels at different age levels. It was found that $C Q$ decreases in the aging voice. It may be the result of losing elasticity of the ligaments in vocal folds resulting in lower rate of glottis abduction and adduction. Further studies must be required to find significance of $C Q$ values in perspectives of age compared with untrained singers. ${ }^{11}$

Contact index (Singing task): Contact index mean of (0.3583) trained female singers was also higher than $\mathrm{Cl}$ mean $(0.2463)$ of untrained singers in singing task. The results showed that trained 
singers have significantly higher $\mathrm{Cl}$ values for both speech task and singing task. Contact index is defined as the difference between increasing and decreasing of vocal folds contacts in vocal folds vibration. Contact index reflects the symmetry of the contact phase, is thought to reflect vocal fold tonus, and to be particularly sensitive to mucosal dynamics within the vertical plane. ${ }^{12}$

Contact index mean values were slightly higher in the singing task as the participants sung the vowel /a/ and it required more energy. Through the practice of singing singers were able to manipulate glottis abduction and adduction more than untrained singers. It may reflect reasons of higher values of $\mathrm{Cl}$ mean in singing task.

Herbst and Sevec studied on $\mathrm{Cl}$ values between trained singer and nonsingers. Four vowels were sung by their participant in chest and falsetto register. Trained singers showed higher values of $\mathrm{Cl}$ in two types of register. They concluded that exercise enable the singers to separately manipulate cartilaginous adduction and membranous medicalization of the glottis through bulging of vocal folds. which is responsible for higher $\mathrm{Cl}$ values in singers. ${ }^{13}$

Lankeren studied on voice characteristics of amateur female tenors who were comparable with those of male tenors. It was found that all subjects produced $\mathrm{Cl}$ of $60 \%$ or higher in singing with a loud chest voice. There was more variance between the individuals than between groups of male and female singers. Thus the present study assumes that $\mathrm{Cl}$ is a useful parameter of EGG, which can vary in terms of dynamic variation and tonality of the voice. ${ }^{14}$

Contact index (Speech task): Contact index mean (0.2760) of trained female singers was also higher than $\mathrm{Cl}$ mean (0.1143) of untrained singers in speech task. The present study focuses on the significance of $\mathrm{Cl}$ value in terms of vocal folds physiology. Decrease in the $\mathrm{Cl}$ values suggests an asymmetry vocal fold physiological adjustment. ${ }^{15}$ Trained singers' voice has more dynamic variations and tonality than untrained singers' voice. ${ }^{16}$ Thus its is assumed that trained singers have slightly higher $\mathrm{Cl}$ values than do untrained singers.

Increment of the $\mathrm{Cl}$ values was depending upon the amount of exposure and practice strategies of singing. Cecconello (2010) analyzed the significance of $\mathrm{Cl}$ in two groups on the basis of singing experience. ${ }^{10}$ They have found that $\mathrm{Cl}$ values are higher in a group with more singing experiences. Researchers also found $\mathrm{Cl}$ varied with different styles of singing between male and female singing voices. Grawunder (2009) studied on production types of "western" overtone singing and South Siberian throat singing. Result showed that $\mathrm{Cl}$ values varied in the two types of singing. ${ }^{17}$

Contact quotient perturbation (Singing task): Contact quotient perturbation mean (3.028) of trained singers was lower than CQP mean (3.915) of untrained singers in singing task. Perturbation means disturbance or uneasy. Contact quotient perturbation is defined as the amount of the values of vocal folds uneasiness or disturbance in one cycle of glottal contact and opening time. It is the value of occurrences of perturbations in the CQ of vocal folds vibration. Values of perturbation indicate the quality of voice and lower values of perturbation reflect smooth symmetry of vocal folds contacts. ${ }^{18}$

Singing with vowel required more energy and more breath control. Trained singers have a control over their breath than the untrained singers, which is responsible for reducing their perturbations values. Increased vocal efficiency of trained singers in comparison to an untrained one is obvious because of their superior control over breathing and phonation. ${ }^{18}$ Specifically, trained singers have independent pitch and loudness control through regulating subglottal pressure. ${ }^{19}$ Collony et al. studied the effects of vocal training in trained and untrained singers and concluded that classical voice training improves breath control by decreasing the amount of airflow and subglottal pressure necessary to complete difficult singing tasks. ${ }^{20}$ Butte et al. measured perturbation features of EGG and nonlinear dynamics in different singing styles and lowered values of CQP obtained in all types of singing and found that classical singers showed more flexible features of CQP and EGG perturbation compared to semiclassical vocalists. ${ }^{21}$

Contact quotient perturbation (Speech task): Contact quotient perturbation mean (1.733) of trained singers was lower than the CQP mean (2.9546) of untrained singers in speech task. Trained singers have higher $C Q$ values, so the perturbation values must be less in their voice. For this reason, it can be assumed that a singer has more stable and constant vocal folds oscillation with less numbers of perturbation. Mendes et al. studied the effect of training on singing voice with phonating vowels through chest register. ${ }^{22}$ Professional trained singers were trained to distribute efficient air flow in phonation by their training experiences, which was the reason for reduced CQP values further concluded that singers have more stable vocal fold oscillations with reference to of long-term singing experiences. Cecconello, Salvador, and Córdoba obtained lower values of CQP in their group as a consequence of experienced singing. ${ }^{10}$

Contact index perturbation mean (93.89) of trained singers was lower than the CIP mean (103.04) of untrained in singing task. Contact index perturbation is defined as the occurrence of perturbation in a contact phase. Higher values of perturbation is the predominant index of vocal folds contact asymmetry. ${ }^{15}$ Trained singers showed acceptable symmetry of vocal folds with reference to contact phase than the untrained singers. Perturbation values are found more in the singing task. Higher perturbation values were found to be increased in singing task compare to speech task. It would be further concluded that perturbation values were increased with the variation of vocal folds contacts.

Professionally trained singers have significant control power in modulating vocal tone. Many singers demonstrated improved vocal tone production created through the efficient use of vocal apertures. Dejonckere et al. studied the effects of music education on children's voice. Lower values of CIP obtained in singing task for those children who exposed to vocal exercises. Study was inferred that vocal exercises help to modulate tone with less amount of perturbation. ${ }^{23}$

Contact index perturbation (Speech task): Contact index perturbation mean (93.69) was lower than 30 untrained singers (CIP mean $=71.78)$ in speech task. Contact index perturbation values measured smoothness or regularity of vocal folds. Gould and coworkers analyzed CIPCIP in sustained phonation task in normal subjects and pathological subjects and CIPs were found higher in pathological groups. Very high CIP value was indicative of irregularity of vocal folds for pathological groups. The present study assumed that trained singers had more regularity of vocal fold vibratory patterns than untrained singers. ${ }^{24}$ Singers may be taught to condition over the vocal tone production errors throughout their practices. According to another research by Cecconello, Salvador, and Córdoba low values of CIP were obtained in the group with more signing experiences. Studies have been focused on how CIP values related with vocal effort levels. ${ }^{10}$ 
Huang, Minifie, Kasuya and Lin (1995) measured CIP values while comparing three vocal efforts namely low, normal, and high, respectively. Contact index values were varied significantly among the three vocal effort levels, where CIP values were lowered in low vocal efforts. ${ }^{25}$

Contact ratio (Singing task): Contact ratio mean (63.816) of trained female singers was also higher than CR mean (59.988) of untrained female singers in singing task. Contact ratio is defined as the ratio of the glottal closed time and open time over fundamental frequency. The present study assumed from the above results that the amount of vocal folds contact was very high in trained singers and may be explained in terms of laryngeal physiology. Singers stored less amount of air in each cycle of vibration that increased the time of glottal closure and opening so that the $C R$ values are increased. ${ }^{8}$ The trained singer used a technique to close the vocal folds. This technique is often referred as a closed-throat technique or high-larynx technique for which voice is produced without producing excess laryngeal pressure and vocal folds opening and closing without too much tension. ${ }^{26}$

Wang and Zhang measured CR in acoustic test of vowels like /e/, /a:/, /o/, which were sung by 10 opera singers and measured through Dr. Speech version IV software and significant differences of $C R$ values were obtained. ${ }^{9}$

Contact ratio (Speech task): Conatct ratio mean (55.29) of trained female singers was also higher than CR mean (46.46) of untrained female singers in speech task. Contact ratio values in phonation task can be regulated by laryngeal adjustment. ${ }^{8}$ Values of vocal folds contact depend on two factors (1) abduction or adduction of the vocal folds and (2) stiffness and thickness of the vocal folds. ${ }^{27}$ Mecke et al. measured CR values in children's singing voice in speech task. Higher values of CR were obtained in phonation task. It was concluded that the effect of training is influenced by the rate of glottis opening and closing in children's voice. Studies have been carried out to focus how $C R$ values are related with singing experiences. Contact ratio is associated with training of efficiency in vocal folds. ${ }^{28}$ Cecconello, Salvador, and Córdoba analyzed the efficiency of qualitative and quantitative parameters of the EGG in order to differentiate among singers with different singing styles and experiences. They have found higher CR values in their groups of higher singing experiences. ${ }^{10}$

\section{Conclusion}

Electroglottography parameters were well-taken measures, which can reflect the training aspects of singing in a very much sensitive way. The present study attempted to find out significance of electroglottographical parameterization in female trained (Hindustani singer) and untrained singers. It was necessary to determine the effect of training on each parameters of electroglottograph. Trained singers were considered subjects having formal training in vocal music for a period of more than 5 years and untrained subjects having no exposure to formal training in vocal music. ${ }^{29}$ The results show the usefulness of qualitative aspects of the EGG parameters to differentiate the vocal function altered by the glottal vibratory characteristics in trained singers, from the untrained female singers. All the parameters are proved to be useful to show differences. The quantitative parameters with higher significance to differentiate the two study groups were $\mathrm{CQ}, \mathrm{Cl}, \mathrm{CQP}, \mathrm{CIP}$, and $\mathrm{CR}$, which provide information with respect to the glottis contact, the vibratory regularity, the vibration symmetry, and the speed of closing and opening of the vocal folds.

\section{IMPLICATION}

This study implicates the most significant parameters of trained and untrained singers and establishes a valid norm for references in terms of normative. Thus if any pathological cases it can be well assumed that the amount of changes possible to make the vocal pathogenesis of two groups of singers mainly trained and untrained singer.

The study would help professional voice users to understand the impact of training on their phonatory capability and on their everyday speech pattern, thereby providing vocal education to the singers to prevent vocal abuse specially in case of untrained singers.

\section{ORCID}

Joyanta CMandal ๑ https://orcid.org/0000-0001-8233-2313

\section{REFERENCES}

1. Åkerlund L, Gramming P. Average loudness level, mean fundamental frequency, and subglottal pressure: comparison between female singers and nonsingers. J Voice 1994;8(3):263-270. DOI: 10.1016/s0892-1997(05)80298-x

2. Awan SN. Phonetographic profiles and FO-SPL characteristics of untrained versus trained vocal groups. J Voice 1991;5(1):41-50. DOI: 10.1016/S0892-1997(05)80162-6

3. Ghosh, K. Comparison of acoustic characteristics in female trained (Carnatic style) singers, untrained singers and non singers (Unpublished Master's thesis). University of Mysore.

4. Miller DG, Schutte HK. Physical definition of the "flageolet register". J Voice 1993;7(3):206-212. DOI: 10.1016/S0892-1997(05)80328-5

5. Higgins MB, McCleary EA, Ide-Helvie DL, Carney AE. Speech and voice physiology of children who are hard of hearing. Ear Hear 2005;26(6):546-558. DOI: 10.1097/01.aud.0000188151.99086.a3

6. Rothenberg M, Mahshie JJ. Monitoring vocal fold abduction through vocal fold contact area. J Speech Hear Res 1988;31(3):338-351. DOI: 10.1044/jshr.3103.338

7. Rose A, Jokhovurd RD. Electroglottography analysis of vocal technique in singers voice Arch Otolaryngol 1988;35:335-398.

8. Howard DM. Variation of electrolaryngographically derived closed quotient for trained and untrained adult female singers. J Voice. 1995;9(2):163-172. DOI: 10.1016/s0892-1997(05)80250-4

9. Wang J, Zhang Q. Analysis and study on electroglottograph and acoustic parameters of peking Opera singers. The Voice Foundation's 38th Annual Symposium: Care of the Professional Voice. Westin Philadelphia, Pennsylvania, USA; 2004

10. Cecconello LA. Qualitative and quantitative aspects of the electroglottography in singers with different glottic contact patterns. In28th World Congress of the International Association of Logopedics and Phoniatrics 2010 (pp. 779-782). Sanatorio del Salvador, Córdoba, Argentina.

11. Bier SD, Watson CI. Electroglottographic Analysis of Young and Old Speakers of New Zealand English'. In14th Australasian International Conference on Speech Science and Technology 2012 (pp. 65-69).

12. Orlikoff RF. Vocal stability and vocal tract configuration: an acoustic and electroglottographic investigation. J Voice 1995;9(2):173-181. DOI: 10.1016/s0892-1997(05)80251-6

13. Herbst CT, Svec JG. Membranouss and cartilaginous vocal folds adduction in singing. J Acoustic Soc Am 2012;129:2253-2262. DOI: $10.1121 / 1.3552874$

14. van Lankeren $\mathrm{H}$. Voice characteristics of amateur female tenors are comparable with those of male tenors. Logoped Phoniatr Vocol 2010;35(1):51-58. DOI: 10.3109/14015431003667660 
15. Orlikoff RF. Assessment of the dynamics of vocal fold contact from the electroglottogram: data from normal male subjects. J Speech Hear Res 1991;34(5):1066-1072. DOI: 10.1044/jshr.3405.1066

16. Miller DG. Registers in singing: empirical and systematic studies in the theory of the singing voice. Rijksuniversiteit Groningen; 2000.

17. Grawunder S. On the physiology of voice production in SouthSiberian throat singing: analysis of acoustic and electrophysiological evidences. Frank \& Timme GmbH. 2009.

18. Herbst CT, Ternström S, Švec JG. Investigation of four distinct glottal configurations in classical singing-a pilot study. J Acoust Soc Am 2009;125(3):EL104-EL109. DOI: 10.1121/1.3057860

19. Sundberg J. Perceptual aspects of singing. J Voice 1994;8(2):106-122. DOI: 10.1016/s0892-1997(05)80303-0

20. Collony T, Bridget WK, Russel J. Singing education effects on electroglottography. Logopedphonatir Vocol 1992;24(4): 178-186.

21. Butte CJ, Zhang $Y$, Song $H$, Jiang JJ. Perturbation and nonlinear dynamic analysis of different singing styles. J Voice 2009;23(6): 647-652. DOI: 10.1016/j.jvoice.2008.02.004

22. Mendes AP, Brown Jr WS, Rothman HB, Sapienza C. Effects of singing training on the speaking voice of voice majors. J Voice 2004;18(1):83-89. DOI: 10.1016/j.jvoice.2003.07.006
23. Dejonckere PH, Wieneke GH, Bloemenkamp D, Lebacq J. Fo-perturbation and Fo/loudness dynamics in voices of normal children, with and without education in singing. Int J Pediatr Otorhinolaryngol 1996;35(2):107-115. DOI: 10.1016/0165-5876(95)01291-5

24. Haji T, Horiguchi S, Baer T, Gould WJ. Frequency and amplitude perturbation analysis of electroglottograph during sustained phonation. J Acoust Soc Am 1986 Jul;80(1):58-62. DOI: $10.1121 / 1.394083$

25. Huang DZ, Minifie FD, Kasuya H, Lin SX. Measures of vocal function during changes in vocal effort level. J Voice 1995;9(4):429-438. DOI: 10.1016/s0892-1997(05)80206-1

26. Karyn N. Vibrato: what it is and how to develop it. J Singing 2010;12(3):143-152. https://www.singwise.com/articles/vibrato-whatit-is-and-how-to-develop-it

27. Hirose $\mathrm{H}$. Investigating the physiology of laryngeal structures. The Handbook of Phonetic Sciences, Vol. 2. 2010. p. 130-152.

28. Mecke AC, Sundberg J, Granqvist S, Echternach M. Comparing closed quotient in children singers' voices as measured by high-speed-imaging, electroglottography, and inverse filtering. J Acoust Soc Am 2012;131(1):435-441. DOI: 10.1121/1.3662061

29. Sheela EV. Comparative study of vocal parameters of trained and untrained singers (Unpublished Masters Dissertation). Mysore: University of Mysore; 1974. 